\title{
On the $p$ th moment estimates for the solution of stochastic differential equations
}

\author{
Young-Ho Kim*
}

Dedicated to Professor Shih-sen Chang on his 80th birthday.

"Correspondence:

yhkim@changwon.ac.kr Department of Mathematics,

Changwon National University,

Changwon, 641-773, Republic of Korea

\begin{abstract}
The main aim of this paper is to discuss the $L^{p}$-estimate of solution of stochastic differential equations under nonlinear growth condition. Furthermore, almost surely asymptotic estimates for the solutions of these equations are given. More accurately, we shall estimate sample Lyapunov exponent almost surely.

MSC: $60 \mathrm{H} 10 ; 60 \mathrm{H} 30$

Keywords: Itô's formula; $L^{p}$-estimate; almost surely asymptotic estimates; sample Lyapunov exponent
\end{abstract}

\section{Introduction}

Stochastic analysis including Brownian motion processes has played an important role in many areas of science and engineering for a long time. Since the white noise is mathematically represented by a formal derivative of a Brownian motion process, such stochastic analysis is based on various types of stochastic differential equations(SDEs) of Itô type as a stochastic model. After Itô introduced his stochastic calculus, the theory of SDEs has been developed very quickly. SDEs is the most fundamental concept in modern stochastic models.

Consequently, there is an increasing interest in SDEs. The main interest in the field has often referred to the existence and uniqueness of solutions as well as to study of their qualitative and quantitative properties, with a special emphasis on the analysis of various types of $L^{p}$-estimate, almost sure estimate, stability and approximation. We refer the reader to monographs [1] by Henderson and Plaschko, [2] by Kolmanovskii and Myshkis, and [3] by Mao, among others, and the literature cited therein.

It is well known that the problems of the solutions to SDEs have received considerable attention from the theoretical point of view. One of the classical results and a subject for inquiry in the study of SDEs is an existence and uniqueness theorem of the solution to SDEs under some special conditions. For the work on the existence and uniqueness theorem of the SDEs, we refer the reader to [4] by Cho et al., [5] by Ren and Xia, [6] by Wei and Wang, and [3] by Mao. Now there is an extensive literature discussing SDEs with $p$ th moment estimate and stability of the solution (see [7] by Govindan, [8] by Kim, [9] by Li and $\mathrm{Fu}$, and [10] by Mao et al.).

Further, in the study of the solution for the SDEs, one question arises naturally: Does the $p$ th moment of the solution assure the solution for such SDEs? To the best of our knowl-

○2014 Kim; licensee Springer. This is an Open Access article distributed under the terms of the Creative Commons Attribution License (http://creativecommons.org/licenses/by/2.0), which permits unrestricted use, distribution, and reproduction in any medium, provided the original work is properly cited. 
edge, there are few results on this problem. It is also worth noting that the $p$ th moment of the solution for such SDEs has not been fully investigated, which remains an interesting research topic.

Our study is essentially based on [3] by Mao referring to the $L^{p}$-estimate and almost surely asymptotic estimates of SDEs. In fact, we generalize Mao's results by finding sufficient conditions, which are easy to verify, guaranteeing $L^{p}$-estimate and almost surely asymptotic estimates of the solutions of these equations.

\section{Preliminary}

Let $|\cdot|$ denote an Euclidean norm in $R^{n}$. If $A$ is a vector or a matrix, its transpose is denoted by $A^{T}$; if $A$ is a matrix, its trace norm is represented by $|A|=\sqrt{\operatorname{trace}\left(A^{T} A\right)}$. Let $t_{0}$ be a positive constant and $(\Omega, \mathcal{F}, P)$ throughout this paper, unless otherwise specified, be a complete probability space with a filtration $\left\{\mathcal{F}_{t}\right\}_{t \geq t_{0}}$ satisfying the usual conditions (i.e., it is right continuous and $\mathcal{F}_{t_{0}}$ contains all $P$-null sets). Assume that $B(t)$ is an $m$-dimensional Brownian motion defined on a complete probability space, that is, $B(t)=\left(B_{1}(t), B_{2}(t), \ldots, B_{m}(t)\right)^{T}$. Let $\mathcal{L}^{p}\left([a, b] ; R^{d}\right)$ denote the family of $R^{d}$-valued $\mathcal{F}_{t^{-}}$ adapted processes $\{f(t)\}_{a \leq t \leq b}$ such that $\int_{a}^{b}|f(t)|^{p} d t<\infty$ a.s.

Consider the $d$-dimensional stochastic differential equations of Itô type

$$
d x(t)=f(x(t), t) d t+g(x(t), t) d B(t) \quad \text { on } t_{0} \leq t \leq T
$$

with initial value $x\left(t_{0}\right)=x_{0}, f: R^{d} \times\left[t_{0}, T\right] \rightarrow R^{d}$, and $g: R^{d} \times\left[t_{0}, T\right] \rightarrow R^{d \times m}$ be Borel measurable.

In [3], the author presented a result stating that, for initial value $x\left(t_{0}\right)=x_{0}$, the $p$ th moment of the unique solution $x(t), t_{0} \leq t \leq T$ of equation (2.1) under Lipschitz condition and linear growth condition will grow at most exponentially with exponent $p \alpha$. We reproduce the result here.

Theorem 2.1 Let $p \geq 2$ and $x_{0} \in L^{p}\left(\Omega ; R^{d}\right)$. Assume that there exists a constant $\alpha$ such that for all $(x, t) \in R^{d} \times\left[t_{0}, T\right]$,

$$
x^{T} f(x, t)+\frac{p-1}{2}|g(x, t)|^{2} \leq \alpha\left(1+|x|^{2}\right) .
$$

Then

$$
E|x(t)|^{p} \leq 2^{(p-2) / 2}\left[1+E\left|x_{0}\right|^{p}\right] \exp \left(p \alpha\left(t-t_{0}\right)\right) .
$$

\section{Main results}

The topic of our analysis is equation (2.1) with initial data $x\left(t_{0}\right)=x_{0}$. An $\left\{\mathcal{F}_{t}\right\}$-adapted process $x(t)$ with values in $R^{d}$ is said to be the solution to equation (2.1) if it satisfies the initial condition and the corresponding stochastic integral equation holds a.s., i.e., for every $t \geq t_{0}$

$$
x(t)=x_{0}+\int_{t_{0}}^{t} f(x(s), s) d s+\int_{t_{0}}^{t} g(x(s), s) d B(s) \quad \text { on } t_{0} \leq t \leq T .
$$


The basic existence and uniqueness theorem based on the Picard method of iterations requires the global or, in a weakened version, Lipschitz condition and linear growth condition for $f$ and $g$ (see [3-6]). Then there exists a unique a.s. continuous and adapted solution $x(t)$ to equation (2.1) satisfying $E\left|\sup _{t_{0} \leq t \leq T} x(t)\right|^{2}<\infty$ for every $T \geq t_{0}$ under the linear growth condition. Moreover, if $x_{0} \in L^{p}\left(\Omega ; R^{d}\right), p \geq 2$, then $E|x(t)|^{p}<\infty$ under the monotone condition. Since our goal is to study $L^{p}$-estimate and almost surely asymptotic estimate problems, we assume that there exists a unique solution $x(t)$ to equation (2.1) under non-Lipschitz condition and nonlinear growth condition (see $[5,6])$. We also assume that all the Lebesgue and Itô's integrals employed further are well defined.

We start with the following $L^{p}$-estimate.

Theorem 3.1 Let $p \geq 2$ and $x_{0} \in L^{p}\left(\Omega ; R^{d}\right)$. Assume that for all $(x, t) \in R^{d} \times\left[t_{0}, T\right]$, $\{f(x(t), t)\} \in \mathcal{L}^{1}\left(\left[t_{0}, T\right] ; R^{d}\right)$, and $\{g(x(t), t)\} \in \mathcal{L}^{2}\left(\left[t_{0}, T\right] ; R^{d}\right)$, it follows that

$$
x^{T} f(x(t), t) \vee \frac{p-1}{2}|g(x(t), t)|^{2} \leq \kappa\left(1+|x(t)|^{2}\right),
$$

where $\kappa(\cdot)$ is a concave nondecreasing function from $R_{+}$to $R_{+}$such that $\kappa(0)=0, \kappa(u)>0$ for $u>0$. Then, for a pair of positive constants $a$ and $b$ such that $\kappa(u) \leq a+b u$, we have

$$
E|x(t)|^{p} \leq 2^{(p-2) / 2}\left[1+E\left|x_{0}\right|^{p}\right] \exp \left(2 p(a+b)\left(t-t_{0}\right)\right)
$$

for all $t \in\left[t_{0}, T\right]$.

Proof By Itô's formula, we can derive that for $t \in\left[t_{0}, T\right]$,

$$
\begin{aligned}
{\left[1+|x(t)|^{2}\right]^{p / 2}=} & {\left[1+\left|x\left(t_{0}\right)\right|^{2}\right]^{p / 2}+p \int_{t_{0}}^{t}\left[1+|x(s)|^{2}\right]^{(p-2) / 2} x^{T}(s) f(x(s), s) d s } \\
& +\frac{p}{2} \int_{t_{0}}^{t}\left[1+|x(s)|^{2}\right]^{(p-2) / 2}|g(x(s), s)|^{2} d s \\
& +\frac{p(p-2)}{2} \int_{t_{0}}^{t}\left[1+|x(s)|^{2}\right]^{(p-4) / 2}\left|x^{T}(s) g(x(s), s)\right|^{2} d s \\
& +p \int_{t_{0}}^{t}\left[1+|x(s)|^{2}\right]^{(p-2) / 2} x^{T}(s) g(x(s), s) d B(s) .
\end{aligned}
$$

By condition (3.1), it is easy to see that

$$
\begin{aligned}
{\left[1+|x(t)|^{2}\right]^{\frac{p}{2}} \leq } & 2^{\frac{p-2}{2}}\left(1+\left|x_{0}\right|^{p}\right)+2 p \int_{t_{0}}^{t}\left[1+|x(s)|^{2}\right]^{\frac{p-2}{2}} \kappa\left(1+|x(s)|^{2}\right) d s \\
& +p \int_{t_{0}}^{t}\left[1+|x(s)|^{2}\right]^{(p-2) / 2} x^{T}(s) g(x(s), s) d B(s) .
\end{aligned}
$$

Given that $\kappa(\cdot)$ is concave and $\kappa(0)=0$, we can find a pair of positive constants $a$ and $b$ such that $\kappa(u) \leq a+b u$ for all $u \geq 0$. And for each number $n \geq 1$, define the stopping time

$$
\tau_{n}=T \wedge \inf \left\{t \in\left[t_{0}, T\right]:|x(t)| \geq n\right\} .
$$


Obviously, as $n \rightarrow \infty, \tau_{n} \uparrow T$ a.s. Moreover, it follows from (3.3) and the property of Itô's integral that

$$
\begin{aligned}
E\left(\left[1+\left|x\left(t \wedge \tau_{n}\right)\right|^{2}\right]^{\frac{p}{2}}\right) & \leq 2^{\frac{p-2}{2}}\left[1+E\left|x_{0}\right|^{p}\right]+2 p(a+b) E \int_{t_{0}}^{t \wedge \tau_{n}}\left[1+|x(s)|^{2}\right]^{\frac{p}{2}} d s \\
& \leq 2^{\frac{p-2}{2}}\left[1+E\left|x_{0}\right|^{p}\right]+2 p(a+b) \int_{t_{0}}^{t} E\left(\left[1+\left|x\left(s \wedge \tau_{n}\right)\right|^{2}\right]^{\frac{p}{2}}\right) d s .
\end{aligned}
$$

The Gronwall inequality yields

$$
E\left(\left[1+\left|x\left(t \wedge \tau_{n}\right)\right|^{2}\right]^{\frac{p}{2}}\right) \leq 2^{\frac{p-2}{2}}\left[1+E\left|x_{0}\right|^{p}\right] e^{2 p(a+b)\left(t-t_{0}\right)} .
$$

Letting $n \rightarrow \infty$ yields

$$
E\left(\left[1+|x(t)|^{2}\right]^{\frac{p}{2}}\right) \leq 2^{\frac{p-2}{2}}\left[1+E\left|x_{0}\right|^{p}\right] e^{2 p(a+b)\left(t-t_{0}\right)}
$$

and the desired inequality follows.

Let us now turn to considering the case of $0<p<2$. This is rather easy if we note that the Hölder inequality implies

$$
E|x(t)|^{p} \leq\left(E|x(t)|^{2}\right)^{\frac{p}{2}} .
$$

In other words, the estimate for $E|x(t)|^{p}$ can be done via the estimate for the second moment. For instance, we have the following corollary.

Corollary 3.2 Let $0<p<2$ and $x_{0} \in L^{p}\left(\Omega ; R^{d}\right)$. Assume that for all $(x, t) \in R^{d} \times\left[t_{0}, T\right]$, $\{f(x(t), t)\} \in \mathcal{L}^{1}\left(\left[t_{0}, T\right] ; R^{d}\right)$, and $\{g(x(t), t)\} \in \mathcal{L}^{2}\left(\left[t_{0}, T\right] ; R^{d}\right)$, it follows that

$$
x^{T} f(x(t), t) \vee \frac{1}{2}|g(x(t), t)|^{2} \leq \kappa\left(1+|x(t)|^{2}\right),
$$

where $\kappa(\cdot)$ is a concave nondecreasing function from $R_{+}$to $R_{+}$such that $\kappa(0)=0, \kappa(u)>0$ for $u>0$. Then, for a pair of positive constants $a$ and $b$ such that $\kappa(u) \leq a+b u$, we have

$$
E|x(t)|^{p} \leq\left[1+E\left|x_{0}\right|^{2}\right]^{\frac{p}{2}} \exp \left(2 p(a+b)\left(t-t_{0}\right)\right)
$$

for all $t \in\left[t_{0}, T\right]$.

We now verify that if a nonlinear growth condition

$$
|f(x(t), t)|^{2} \vee|g(x(t), t)|^{2} \leq \kappa_{1}\left(1+|x(t)|^{2}\right),
$$

with $\kappa_{1}(\cdot)$ is a concave nondecreasing function from $R_{+}$to $R_{+}$such that $\kappa_{1}(0)=0, \kappa_{1}(u)>0$ for $u>0$ is fulfilled, then condition (3.1) is satisfied with $\kappa(u)=u \sqrt{\kappa_{1}(u)} \vee \frac{p-1}{2} \kappa_{1}(u)$. In fact, using (3.7) and the elementary inequality $2 a b \leq a^{2}+b^{2}$, one can derive that for any $\epsilon>0$,

$$
\begin{aligned}
2 x^{T} f(x, t) & \leq 2|x||f(x, t)|=2(\sqrt{\epsilon}|x|)(|f(x, t)| / \sqrt{\epsilon}) \\
& \leq \epsilon|x|^{2}+\frac{1}{\epsilon}|f(x, t)|^{2} \leq \epsilon|x|^{2}+\frac{1}{\epsilon} \kappa_{1}\left(1+|x(t)|^{2}\right) .
\end{aligned}
$$


Letting $\epsilon=\sqrt{\kappa_{1}\left(1+|x(t)|^{2}\right)}$ yields

$$
2 x^{T} f(x, t) \leq\left(1+|x(t)|^{2}\right) \sqrt{\kappa_{1}\left(1+|x(t)|^{2}\right)} .
$$

Consequently,

$$
x^{T} f(x, t) \vee \frac{(p-1)}{2}|g(x, t)|^{2} \leq\left(1+|x|^{2}\right) \sqrt{\kappa_{1}\left(1+|x|^{2}\right)} \vee \frac{(p-1)}{2} \kappa_{1}\left(1+|x|^{2}\right) .
$$

We therefore obtain the following useful corollary.

Corollary 3.3 Let $p \geq 2$ and $x_{0} \in L^{p}\left(\Omega ; R^{d}\right)$. Assume that the nonlinear growth condition (3.7) holds. Then inequality (3.1) holds with

$$
\kappa\left(1+|x|^{2}\right)=\left(1+|x|^{2}\right) \sqrt{\kappa_{1}\left(1+|x|^{2}\right)} \vee \frac{(p-1)}{2} \kappa_{1}\left(1+|x|^{2}\right) .
$$

We now apply this result to show one of the important properties of the solution.

Theorem 3.4 Let $p \geq 2$ and $x_{0} \in L^{p}\left(\Omega ; R^{d}\right)$. Assume that the nonlinear growth condition (3.7) holds for all $(x, t) \in R^{d} \times\left[t_{0}, T\right]$. Then

$$
E|x(t)-x(s)|^{p} \leq\left[\frac{1}{2} C(2 \alpha)^{\frac{p}{2}}+C 2^{p-2} \beta^{\frac{p}{2}}\left[1+E\left|x_{0}\right|^{p}\right] e^{2 p(a+b)\left(T-t_{0}\right)}\right](t-s)^{\frac{p}{2}},
$$

where $C=\left(2\left(T-t_{0}\right)\right)^{\frac{p}{2}}+\frac{1}{2}(2 p(p-1))^{\frac{p}{2}}$. In particular, the pth moment of the solution is continuous on $\left[t_{0}, T\right]$.

Proof Applying the elementary inequality $|a+b|^{p} \leq 2^{p-1}\left(|a|^{p}+|b|^{p}\right)$, we can easily see that

$$
E|x(t)-x(s)|^{p} \leq 2^{p-1} E\left|\int_{s}^{t} f(x(r), r) d r\right|^{p}+2^{p-1} E\left|\int_{s}^{t} g(x(r), r) d B(r)\right|^{p} .
$$

Using the Hölder inequality, the moment inequality ([3], Theorem 1.7.1), and condition (3.7), one can show that

$$
\begin{aligned}
E|x(t)-x(s)|^{p} \leq & (2(t-s))^{p-1} E \int_{s}^{t}|f(x(r), r)|^{p} d r \\
& +\frac{1}{2}(2 p(p-1))^{\frac{p}{2}}(t-s)^{\frac{p-2}{2}} E \int_{s}^{t}|g(x(r), r)|^{p} d r \\
\leq & C(t-s)^{\frac{p-2}{2}} E \int_{s}^{t}\left(\kappa_{1}\left(1+|x(r)|^{2}\right)\right)^{\frac{p}{2}} d r,
\end{aligned}
$$

where $C=\left(2\left(T-t_{0}\right)\right)^{\frac{p}{2}}+\frac{1}{2}(2 p(p-1))^{\frac{p}{2}}$. Given that $\kappa_{1}(\cdot)$ is concave and $\kappa_{1}(0)=0$, we can find a pair of positive constants $\alpha$ and $\beta$ such that $\kappa_{1}(u) \leq \alpha+\beta u$ for all $u \geq 0$. So we have

$$
E|x(t)-x(s)|^{p} \leq \frac{1}{2} C(2 \alpha)^{\frac{p}{2}}(t-s)^{\frac{p}{2}}+\frac{1}{2} C(2 \beta)^{\frac{p}{2}}(t-s)^{\frac{p-2}{2}} \int_{s}^{t} E\left(1+|x(r)|^{2}\right)^{\frac{p}{2}} d r .
$$


Substituting this into (3.4) yields that

$$
E|x(t)-x(s)|^{p} \leq\left(\frac{1}{2} C(2 \alpha)^{\frac{p}{2}}+C 2^{p-2} \beta^{\frac{p}{2}}\left[1+E\left|x_{0}\right|^{p}\right] e^{2 p(a+b)\left(T-t_{0}\right)}\right)(t-s)^{\frac{p}{2}},
$$

which is the required inequality.

Theorem 3.5 Let $p \geq 2$ and $x_{0} \in L^{p}\left(\Omega ; R^{d}\right)$. Assume that the nonlinear growth condition (3.7) for all $(x, t) \in R^{d} \times\left[t_{0}, T\right]$. Then

$$
E\left(\sup _{t_{0} \leq s \leq t}|x(s)|^{p}\right) \leq C_{2} e^{C_{1} 2^{p-2} \beta^{\frac{p}{2}}\left(t-t_{0}\right)}
$$

for all $t_{0} \leq t \leq T$, where $C_{1}=3^{p-1}\left(T-t_{0}\right)^{\frac{p-2}{2}}\left[\left(T-t_{0}\right)^{\frac{p}{2}}+\left(\frac{p^{3}}{2(p-1)}\right)^{\frac{p}{2}}\right]$ and $C_{2}=1+3^{p-1} E\left|x_{0}\right|^{p}+$ $\frac{1}{2} C_{1}(2 \alpha)^{\frac{p}{2}}\left(T-t_{0}\right)$.

Proof Using the Hölder inequality, the moment inequality ([3], Theorem 1.7.2), and condition (3.7), one can show that

$$
E\left(\sup _{t_{0} \leq s \leq t}|x(s)|^{p}\right) \leq 3^{p-1} E\left|x_{0}\right|^{p}+C_{1} E \int_{t_{0}}^{t}\left(\kappa_{1}\left(1+|x(s)|^{2}\right)\right)^{\frac{p}{2}} d s
$$

where $C_{1}=3^{p-1}\left(T-t_{0}\right)^{\frac{p-2}{2}}\left[\left(T-t_{0}\right)^{\frac{p}{2}}+\left(p^{3} / 2(p-1)\right)^{\frac{p}{2}}\right]$. From the definition of $\kappa_{1}(\cdot)$, we can find a pair of positive constants $\alpha$ and $\beta$ such that $\kappa_{1}(u) \leq \alpha+\beta u$ for all $u \geq 0$. So we have

$$
E\left(\sup _{t_{0} \leq s \leq t}|x(s)|^{p}\right) \leq 3^{p-1} E\left|x_{0}\right|^{p}+\frac{1}{2} C_{1}(2 \alpha)^{\frac{p}{2}}\left(T-t_{0}\right)+C_{1} 2^{p-1} \beta^{\frac{p}{2}} E \int_{t_{0}}^{t}\left(1+|x(s)|^{p}\right) d s .
$$

Hence

$$
1+E\left(\sup _{t_{0} \leq s \leq t}|x(s)|^{p}\right) \leq C_{2}+C_{1} 2^{p-1} \beta^{\frac{p}{2}} \int_{t_{0}}^{t} E\left(1+\sup _{t_{0} \leq r \leq s}|x(r)|^{p}\right) d s
$$

By the Gronwall inequality one can see that

$$
1+E\left(\sup _{t_{0} \leq s \leq t}|x(s)|^{p}\right) \leq C_{2} e^{C_{1} 2^{p-1} \beta^{\frac{p}{2}}\left(t-t_{0}\right)},
$$

which is the required inequality.

Let us now consider a $d$-dimensional stochastic differential equation

$$
d x(t)=f(x(t), t) d t+g(x(t), t) d B(t)
$$

on $t \in\left[t_{0}, \infty\right)$ with initial value $x\left(t_{0}\right)=x_{0} \in L^{2}\left(\Omega, R^{d}\right)$. Assume that the equation has a unique global solution $x(t)$ on $t \in\left[t_{0}, \infty\right)$. Moreover, we shall impose the condition: There is a concave nondecreasing function $\kappa(\cdot)$ from $R_{+}$to $R_{+}$such that, for all $(x, t) \in R^{d} \times$ $\left[t_{0}, \infty\right)$,

$$
x^{T} f(x(t), t) \vee \frac{1}{2}|g(x(t), t)|^{2} \leq \kappa\left(1+|x(t)|^{2}\right) .
$$


Let $0<p \leq 2$. In view of Theorem 3.1 and Corollary 3.2, we know that the $p$ th moment of the solution satisfies

$$
E|x(t)|^{p} \leq\left[1+E\left|x_{0}\right|^{2}\right]^{\frac{p}{2}} \exp \left(2 p(a+b)\left(t-t_{0}\right)\right)
$$

for all $t \geq t_{0}$. This means that the $p$ th moment will grow at most exponentially with exponent $2 p(a+b)$. This can also be expressed as

$$
\limsup _{t \rightarrow \infty} \frac{1}{t} \log \left(E|x(t)|^{p}\right) \leq 2 p(a+b)
$$

The left-hand side of (3.10) is called the pth moment Lyapunov exponent, and (3.10) shows that the $p$ th moment Lyapunov exponent should not be greater than $2 p(a+b)$. In what follows, we shall establish the asymptotic estimate for the solution almost surely. More accurately, we shall estimate

$$
\limsup _{t \rightarrow \infty} \frac{1}{t} \log |x(t)|
$$

almost surely, which is called the sample Lyapunov exponent.

Theorem 3.6 Under condition (3.9), the sample Lyapunov exponent of the solution of equation (3.8) should not be greater than $2(a+b)$, that is,

$$
\limsup _{t \rightarrow \infty} \frac{1}{t} \log |x(t)| \leq 2(a+b)
$$

almost surely.

Proof By Itô’s formula and condition (3.9), we obtain

$$
\begin{aligned}
& \log \left(1+|x(t)|^{2}\right) \\
& =\log \left(1+\left|x_{0}\right|^{2}\right)+2 \int_{t_{0}}^{t} \frac{1}{1+|x(s)|^{2}}\left(x^{T}(s) f(x(s), s)+\frac{1}{2}|g(x(s), s)|^{2}\right) d s \\
& \quad-2 \int_{t_{0}}^{t} \frac{\left|x^{T}(s) g(x(s), s)\right|^{2}}{\left(1+|x(s)|^{2}\right)^{2}} d s+M(t) \\
& \leq \log \left(1+\left|x_{0}\right|^{2}\right)+4 \int_{t_{0}}^{t} \frac{\kappa\left(1+|x(s)|^{2}\right)}{1+|x(s)|^{2}} d s-2 \int_{t_{0}}^{t} \frac{\left|x^{T}(s) g(x(s), s)\right|^{2}}{\left(1+|x(s)|^{2}\right)^{2}} d s+M(t),
\end{aligned}
$$

where

$$
M(t)=2 \int_{t_{0}}^{t} \frac{x^{T}(s) g(x(s), s)}{1+|x(s)|^{2}} d B(s) .
$$

Furthermore, for every integer $n \geq t_{0}$, using the exponential martingale inequality (see [3], Theorem 1.7.4) on sees that

$$
P\left\{\sup _{t_{0} \leq t \leq n}\left[M(t)-2 \int_{t_{0}}^{t} \frac{\left|x^{T}(s) g(x(s), s)\right|^{2}}{\left(1+|x(s)|^{2}\right)^{2}} d s\right]>2 \log n\right\} \leq \frac{1}{n^{2}} .
$$


An application of the Borel-Cantelli lemma then yields that for almost all $\omega \in \Omega$, there is a random integer $n_{0}=n_{0}(\omega) \geq t_{0}+1$ such that

$$
\sup _{t_{0} \leq s \leq t}\left[M(t)-2 \int_{t_{0}}^{t} \frac{\left|x^{T}(s) g(x(s), s)\right|^{2}}{\left(1+|x(s)|^{2}\right)^{2}} d s\right] \leq 2 \log n
$$

if $n \geq n_{0}$. That is,

$$
M(t) \leq 2 \log n+2 \int_{t_{0}}^{t} \frac{\left|x^{T}(s) g(x(s), s)\right|^{2}}{\left(1+|x(s)|^{2}\right)^{2}} d s
$$

for all $t_{0} \leq t \leq n, n \geq n_{0}$ almost surely. From inequality (3.11) and the definition of the function $\kappa(\cdot)$, we deduce that

$$
\log \left(1+|x(t)|^{2}\right) \leq \log \left(1+\left|x_{0}\right|^{2}\right)+4(a+b)\left(t-t_{0}\right)+2 \log n
$$

for all $t_{0} \leq t \leq n, n \geq n_{0}$ almost surely. Therefore, for almost all $\omega \in \Omega$, if $n \geq n_{0}, n-1 \leq$ $t \leq n$,

$$
\frac{1}{t} \log \left(1+|x(t)|^{2}\right) \leq \frac{1}{n-1}\left[\log \left(1+\left|x_{0}\right|^{2}\right)+4(a+b)\left(t-t_{0}\right)+2 \log n\right]
$$

This implies

$$
\begin{aligned}
\limsup _{t \rightarrow \infty} \frac{1}{t} \log (1+|x(t)|) & \leq \limsup _{t \rightarrow \infty} \frac{1}{2 t} \log \left(1+|x(t)|^{2}\right) \\
& \leq \limsup _{t \rightarrow \infty} \frac{1}{2(n-1)}\left[\log \left(1+\left|x_{0}\right|^{2}\right)+4(a+b)\left(t-t_{0}\right)+2 \log n\right] \\
& =2(a+b)
\end{aligned}
$$

almost surely, which is the required inequality.

\section{Competing interests}

The author declares that they have no competing interests.

\section{Acknowledgements}

This research is financially supported by Changwon National University in 2013-2014.

Received: 30 April 2014 Accepted: 19 September 2014 Published: 16 Oct 2014

\section{References}

1. Henderson, D, Plaschko, P: Stochastic Differential Equations in Science and Engineering. World Scientific, Singapore (2006)

2. Kolmanovskii, VB, Myshkis, A: Applied Theory of Functional Differential Equations. Kluwer Academic, Dordrecht (1992)

3. Mao, X: Stochastic Differential Equations and Applications. Horwood, Chichester (2007)

4. Cho, YJ, Dragomir, SS, Kim, Y-H: A note on the existence and uniqueness of the solutions to SFDEs. J. Inequal. Appl. 2012, $126(2012)$

5. Ren, Y, Xia, N: Existence, uniqueness and stability of the solutions to neutral stochastic functional differential equations with infinite delay. Appl. Math. Comput. 210, 72-79 (2009)

6. Wei, F, Wang, K: The existence and uniqueness of the solution for stochastic functional differential equations with infinite delay. J. Math. Anal. Appl. 331, 516-531 (2007)

7. Govindan, TE: Stability of mild solution of stochastic evolution equations with variable delay. Stoch. Anal. Appl. 21, 1059-1077 (2003)

8. Kim, Y-H: An estimate of the solutions for stochastic functional differential equations. J. Appl. Math. Inform. 29(5-6), 1549-1556 (2011) 
9. $L i, X, F u, X$ : Stability analysis of stochastic functional differential equations with infinite delay and its application to recurrent neural networks. J. Comput. Appl. Math. 234, 407-417 (2010)

10. Mao, $X$, Shen, Y, Yuan, C: Almost surely asymptotic stability of neutral stochastic differential delay equations with Markovian switching. Stoch. Process. Appl. 118, 1385-1406 (2008)

10.1186/1029-242X-2014-395

Cite this article as: Kim: On the $p$ th moment estimates for the solution of stochastic differential equations. Journal of Inequalities and Applications 2014, 2014:395

Submit your manuscript to a SpringerOpen ${ }^{\odot}$ journal and benefit from:

- Convenient online submission

- Rigorous peer review

- Immediate publication on acceptance

- Open access: articles freely available online

- High visibility within the field

- Retaining the copyright to your article

Submit your next manuscript at $\gg$ springeropen.com 